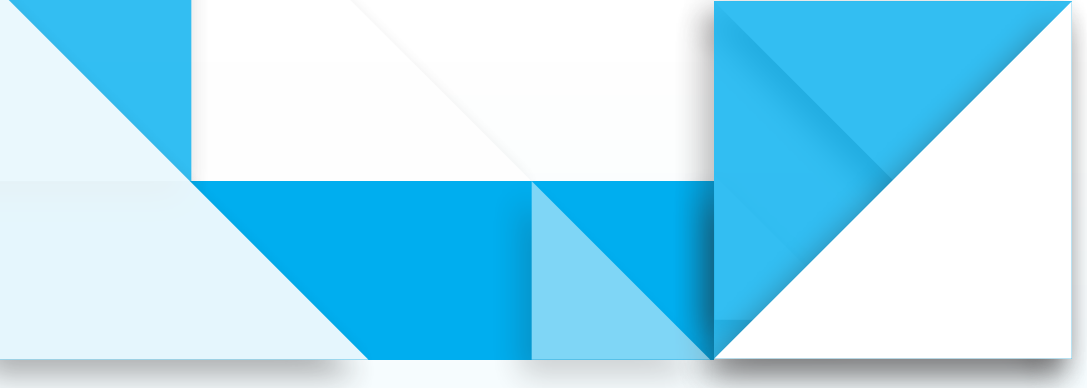

\title{
Los recursos tecnológicos en la estadística bidimensional en los textos españoles de bachillerato
}

\section{María M. Gea* Carmen Batanero** María del Mar López-Martín*** José Miguel Contreras ${ }^{* * *}$}

\section{- Technological Resources in Bidimensional Statistics in High School Textbooks}

- Os recursos tecnológicos na estadística bidimensional nos textos espanhóis do Ensino Médio

\section{Resumen}

Los currículos actuales recomiendan el uso de los recursos tecnológicos en la enseñanza de la estadística, por la ventaja que suponen en el cálculo y representación gráfica, el trabajo con datos reales y el aprendizaje de conceptos a través de la simulación. En esta investigación se analizan los recursos tecnológicos que los libros de texto sugieren para la enseñanza y aprendizaje de la estadística bidimensional en bachillerato (organización y representación de datos bidimensionales, correlación y regresión). La importancia de este aspecto se debe a que extiende la dependencia funcional a situaciones aleatorias y proporciona al alumno oportunidades de modelizar numerosas aplicaciones. Por otro lado, la investigación didáctica muestra dificultades de comprensión y concepciones erróneas en esta área. Mediante un análisis de contenido se han estudiado dieciséis libros de texto españoles, ocho libros de cada una de las dos especialidades de bachillerato que incluyen "Humanidades y Ciencias Sociales" y "Ciencia y Tecnología". En dichos libros se estudia el uso que se hace de recursos tecnológicos en los problemas y procedimientos propuestos, los tipos de recursos que se referencian para trabajar con internet y el contenido de un CD que acompaña a la mayoría de estos textos. Los resultados muestran una escasa presencia de recursos tecnológicos en los textos analizados, a la vez que una gran variabilidad de los recursos descritos. La referencia a recursos en internet se suele restringir a unidades didácticas y no a conjuntos de datos que puedan usarse en proyectos o a simuladores que faciliten la comprensión conceptual. El CD que acompaña a los textos a veces reproduce el mismo texto o se reduce a colecciones de ejercicios tradicionales; son minoría los problemas y procedimientos que usan recursos tecnológicos. Se concluye con algunas recomendaciones para la mejora de estos textos, teniendo en cuenta dichos recursos.

Palabras clave:

Estadística bidimensional, libros de texto, bachillerato, recursos tecnológicos. mmgea@ugr.es, Licenciada en Matemáticas, Licenciado en Estadística, Máster en Didáctica de las Matemáticas, Máster en Estadística Aplicada, Doctor en Didáctica de las Matemáticas. Universidad de Granada, Departamento de Didáctica de la Matemática.

batanero@ugr.es, Licenciada en Matemáticas, Diplomada en Estadística, Doctor en Matemáticas. Universidad de Granada, Departamento de Didáctica de la Matemática. Universidad de Granada, Departamento de Didáctica de la Matemática.

mariadelmarlopez@ugr.es, Licenciada en Matemáticas, Doctor en Economía Aplicada.Licenciada en Matemáticas, Diplomada en Estadística, Doctor en Matemáticas. Universidad de Granada, Departamento de Didáctica de la Matemática.

jmcontreras@ugr.es, Licenciado en Matemáticas, Licenciado en Estadística, Máster en Didáctica de las Matemáticas, Máster en Estadística Aplicada, Doctor en Estadística, Doctor en Didáctica de las Matemáticas. Universidad de Granada, Departamento de Didáctica de la Matemática. 


\begin{abstract}
Current curriculums recommend the use of technological resources in the teaching of statistics, due to the help they represent in the calculation and production of graphs, the work with real data and the learning of several concepts through simulation. In this research, we analyse the different technological resources suggested in high school textbooks for teaching and learning bi-dimensional statistics in high school (organization and representation of bi-dimensional data, correlation and regression). The importance of this issue is due to the fact that it extends functional dependence to random situations and provides students with opportunities to model numerous applications. On the other hand, previous research shows many difficulties of understanding and misconceptions on these topics. The researchers analysed sixteen Spanish text books, using content analysis; eight books of each of the two modalities that include this topic: "Humanities and Social Sciences" and "Science and Technology". In these books was analysed the use of technological resources in the problems and procedures proposed, the references to technological resources in Internet and the content of a CD that supplements most of these textbooks. The results suggest a scarce presence of these resources in the textbooks analysed, and a large variability of the resources described. The reference to Internet resources is often reduced to didactic units and not to data sets that can be used in projects or simulators to facilitate conceptual understanding. The CD which supplements some of these textbooks sometimes reproduces the same text which appears in the book or includes collections of traditional exercises; there are few problems and procedures based on technology. Finally, some recommendations for improvement of these texts are presented, considering technological resources.
\end{abstract}

Keywords:

Bi-dimensional statistics, textbooks, high school, technology.

\title{
Resumo
}

Os currículos atuais sugerem o uso dos recursos tecnológicos no ensino da estadística, pela vantagem que supõe o cálculo, a representação gráfica, o trabalho com dados reais e a aprendizagem através da simulação. Nesta pesquisa são analisados os recursos tecnológicos que os livros de texto sugerem para o ensino e a aprendizagem da estadística no Ensino Médio (organização e representação de dados bidimensionais, correlação e regressão). A importância desses recursos é devida a uma extensão ou ampliação da dependência funcional em termos de situações imprevistas, o que oferece ao aluno oportunidades para a modelagem de varias aplicações. Por outro lado, a pesquisa didática evidencia dificuldades de compreensão e concepções erradas neste tema. Através da análise de conteúdo foram estudados dezesseis livros didáticos espanhóis, oito livros de cada uma das área de Ensino Médio: "Humanidades e Ciências Sociais, Ciência e Tecnologia". Nesses livros estuda-se o uso que se faz dos recursos tecnológicos, os problemas e procedimentos propostos, os tipos de recursos indicados para trabalhar na internet e o conteúdo de um CD anexo aos livros. Os resultados da pesquisa evidenciam a pouca presença dos recursos tecnológicos nos textos analisados, bem como a grande variabilidade dos recursos descritos. Os recursos na internet estão restritos às unidades didáticas e não estão relacionados com os dados que podem usar-se em projetos ou em simulações que facilitem a compreensão conceitual. $\bigcirc C D$ que está junto aos livros as vezes reproduz o mesmo texto do livro ou está reduzido às coleções de atividades tradicionais, são uma minoria de problemas e procedimentos que usam recursos tecnológicos. Conclui-se com algumas recomendações para a melhora desses livros tendo em consideração os recursos tecnológicos.

Palavras-chave:

estadística bidimensional, livros didáticos, Ensino Médio, recursos tecnológico. 


\section{Introducción}

La estadística bidimensional tiene gran relevancia en la formación de los estudiantes de bachillerato, pues pone en juego nuevas heurísticas respecto a sus conocimientos previos sobre la dependencia funcional. Es, además, muy utilizada como herramienta de investigación en la mayoría de las ciencias experimentales y sociales, así como para la predicción, en gestión, política y economía. En consecuencia, es necesario que el estudiante adquiera competencias de tratamiento de los datos bidimensionales mediante el uso de un lenguaje apropiado, y que adquiera significativamente los conceptos de correlación y regresión. También es importante que sea parte de la formación del profesor en línea de lo señalado por Rocha y Gallego (2009).

En el sistema educativo español nos encontramos, en la actualidad, en una modificación curricular en todos los niveles educativos cuya implantación en bachillerato se prevé para el próximo curso escolar 2015-2016 de manera progresiva. En la nueva normativa se mantiene la enseñanza de estadística bidimensional en el primer curso de bachillerato y se explicitan mucho más los contenidos a tratar al respecto, en las dos modalidades en que se enseña matemáticas: "Ciencias" y "Humanidades y Ciencias Sociales". Los contenidos son similares para ambas modalidades, por ejemplo, en la de Humanidades y Ciencias Sociales se indica (MECD, 2015, p. 385):

Estadística descriptiva bidimensional: Tablas de contingencia. Distribución conjunta y distribuciones marginales. Distribuciones condicionadas. Medias y desviaciones típicas marginales y condicionadas. Independencia de variables estadísticas. Dependencia de dos variables estadísticas. Representación gráfica: nube de puntos. Dependencia lineal de dos variables estadísticas. Covarianza y correlación: Cálculo e interpretación del coeficiente de correlación lineal. Regresión lineal. Predicciones estadísticas y fiabilidad de las mismas. Coeficiente de determinación.

Estas indicaciones modifican poco a las vigentes en el presente curso escolar, que son similares en las modalidades en que se enseña matemáticas. Los contenidos fijados son: "Distribuciones bidimensionales. Interpretación de fenómenos sociales y económicos en los que intervienen dos variables a partir de la representación gráfica de una nube de puntos. Grado de relación entre dos variables estadísticas. Regresión lineal. Extrapolación de resultados" (MEC, 2007b, p. 45475).

La importancia y utilidad de este tema para los estudiantes es clara, así como en la mayoría de estudios universitarios; aun así, su enseñanza y aprendizaje no están exentos de problemas didácticos que la investigación, principalmente desde el campo de la psicología y la didáctica, ha descrito. Aquí se destacan, entre otras, las dificultades de comprensión en torno a los conceptos de covarianza y correlación (Estepa y Batanero 1995; Estepa, 2008; Zieffler y Garfield, 2009).

Un recurso para ayudar a los estudiantes a superar muchas de estas dificultades son los recursos tecnológicos. Hoy existe una gran variedad de ellos como la calculadora, hoja de cálculo, applets y programas de ordenador específicos que pueden facilitar la realización de cálculos y gráficos (Pratt, Davies y Connor, 2011 ). El aprendizaje de y a través de recursos tecnológicos es esencial en esta etapa educativa, sobre todo para desarrollar este tema, y se incluye como objetivo general en bachillerato: "Utilizar con solvencia y responsabilidad las tecnologías de la información y la comunicación" (MEC, 2007b, p. 45382). Así 
mismo, en la asignatura de Matemáticas Aplicadas a las Ciencias Sociales I se encontró la siguiente reflexión:

Las herramientas tecnológicas ofrecen la posibilidad de evitar tediosos cálculos que poco o nada aportan al tratamiento de la información, permitiendo abordar con rapidez y fiabilidad los cambiantes procesos sociales mediante la modificación de determinados parámetros y condiciones iniciales. (MEC, 2007b, p. 45474).

Igualmente se recomienda el uso de estos recursos por parte de la Junta de Andalucía, la cual indica como meta: "Formación para la utilización de las tecnologías de la información y la comunicación, estimulando su uso en los procesos de enseñanza y aprendizaje de todas las materias y en el trabajo del alumnado" (Consejería de Educación, 2008, pp. 9-10).

El objetivo de este trabajo es analizar el uso que se propone de recursos tecnológicos en los libros de texto del bachillerato sobre estadística bidimensional. Nos apoyamos en la importancia del libro de texto, que sirve de orientación al docente y al estudio autónomo del estudiante (Cordero y Flores, 2007).

Estos libros se incluyen en el proceso de transposición didáctica, en el cual, el conocimiento matemático formal se adapta para convertirlo en conocimiento matemático para ser enseñado (Chevallard, 1991). Además, su estudio es un componente del análisis curricular, pues el currículo escrito constituye un paso entre el currículo pretendido y el implementado en el aula (Herbel, 2007).

El trabajo forma parte de un proyecto de investigación (Gea, 2014) donde se analizan la correlación y regresión en los libros de texto. Resultados complementarios se han publicado en Gea, Batanero, Cañadas y Contreras (2013); Gea, Batanero, Fernandes y Gómez (2014) y Gea, Batanero, Arteaga, Cañadas y Contreras (2014).

\section{Marco teórico}

\section{Enfoque ontosemiótico}

Nos basamos en el enfoque ontosemiótico (EOS) (Godino, 2002; Godino, Batanero y Font, 2007) que concibe la matemática como quehacer humano, fundamentada en un lenguaje simbólico y lógicamente organizada. Se consideran los siguientes tipos de objetos matemáticos: problemas planteados, lenguaje, procedimientos, argumentos, conceptos y proposiciones o propiedades asociadas. El significado de estos objetos es relativo según sea visto por el sujeto que resuelve la situación problema (significado personal), o desde la institución encargada de albergar dicho objeto matemático (significado institucional) (Godino y Batanero, 1998). También se consideran diversas facetas duales, que aportan sentido a la enseñanza y aprendizaje de este objeto (Godino, Batanero y Font, 2007): a) institucional y personal, ya comentada; b) ostensiva y no ostensiva, según es 
materializado el objeto (representado) o solo imaginado; c) extensiva e intensiva, según una clase de objetos se defina como una colección por enumeración o mediante una propiedad característica; d) unitaria y sistémica, dependiendo de si el objeto se considera indivisible o compuesto por partes, y e) expresión y contenido, que tiene en cuenta la diferencia entre signo o símbolo, y su significado.

Los recursos tecnológicos ayudan a diferenciar y enriquecer estos objetos y facetas; además permiten resolver problemas más complejos y, en contexto real, fomentan en el estudiante el uso del lenguaje (verbal, simbólico, icónico, gráfico), facilitan y amplían la gama de procedimientos a su alcance, hacen ostensivos conceptos y propiedades, sobre todo por medio de la simulación, y promueven el razonamiento y la argumentación. Al manipular objetos ostensivos, el estudiante irá desarrollando la capacidad de anticipación de los resultados e idealizando progresivamente los ejemplos particulares al objeto general no ostensivo. La faceta extensiva e intensiva aparece al generalizar el ejemplo particular con que trabaja el estudiante a un conjunto más amplio de situaciones, el ejemplo en este caso, está ligado también a la dualidad sistémica y unitaria, que se presenta al construir el significado personal a partir de objetos matemáticos previos, que participan con un rol unitario, y que cuando se construyeron tenían un rol sistémico. En últimas, la dualidad personal e institucional la promueve el profesor al institucionalizar los logros del estudiante.

\section{Papel de los recursos tecnológicos en la enseñanza y aprendizaje de la estadística}

El uso de recursos tecnológicos en la enseñanza de la estadística ha sido reconocido, entre otros, por Pratt, Davies y Connor (2011), quienes destacan la reducción del tiempo de cálculo y la ampliación del tipo de gráficos que el alumno puede realizar interactivamente. Igualmente señalan la posibilidad de trabajar con proyectos, en que el alumno parte de un problema de investigación y utilizando conjuntos de datos reales, que hoy día son accesibles desde muchas instituciones en internet, completa todos los pasos de una investigación (Wild y Pfannkuch, 1999).

Una gran ventaja al utilizar estos datos es que se potencia la interdisciplinariedad en clase de estadística, permitiendo aprender contenidos que no se adquieren habitualmente con problemas tomados de los libros de texto; por ejemplo, el efecto de valores atípicos sobre el cálculo de un estadístico (Hall, 2011 1). Al facilitar el cálculo y la representación gráfica, estos recursos disminuyen el problema tradicional en la enseñanza de la estadística en cuanto al desfase entre la comprensión de los conceptos y los medios técnicos de cálculo para poder aplicarlos (Batanero y Díaz, 2011 1).

Otra aplicación característica es la simulación (Biehler, 1997). Las representaciones icónicas de conceptos en la simulación (por ejemplo, la desviación de los puntos de un diagrama de dispersión a la recta de regresión) pueden ayudar a los estudiantes a pensar a través de modelos concretos, cuando todavía no son capaces de generalizar sus ideas matemáticas. Por otro lado, los simuladores facilitan la estimación/predicción o anticipación; y pueden servir para explorar objetos abstractos, creando micromundos virtuales donde los estudiantes pueden experimentar con las diferentes variables que intervienen. Fernandes, Batanero, Contreras y Díaz (2009) añaden el interés de la simulación en el aprendizaje de la modelización, pues una simulación es ya un modelo matemático (al haber simplificado 
la realidad), pero es menos abstracto que el modelo matemático y constituye un puente entre este y la realidad.

\section{Antecedentes}

\section{Investigaciones sobre comprensión de la estadística bidimensional}

Son muchos los estudios relacionados con la comprensión de la estadística bidimensional, que indican que el razonamiento sobre la covariación es una actividad cognitiva fundamental del ser humano (Moritz, 2004; Zieffler, 2006; McKenzie y Mikkelsen, 2007). Parte de estos trabajos detectan sesgos en este razonamiento, como la correlación ilusoria, donde los sujetos crean sus propias teorías sobre la correlación entre dos variables, sin tener en cuenta la correlación real en los datos (Chapman y Chapman, 1967). Otro sesgo muy común es el llamado efecto de la regresión, donde en investigaciones experimentales pueden confundirse efectos del tratamiento con la tendencia de la variable a acercarse a su media, en dos medidas consecutivas de la misma magnitud (Engel y Sedlmeier, 2011).

Por otro lado, Estepa (1994) describe las siguientes concepciones incorrectas sobre la correlación: a) concepción determinista (consistente en aceptar solo la dependencia funcional), b) concepción local (pensar que se puede medir la correlación solo con parte de los datos), unidireccional (no aceptar como correlación la correlación inversa) y c) causal (confundir correlación y causalidad). Estas concepciones permanecen después de la enseñanza, siendo la más resistente al cambio la concepción causal (Batanero, Godino y Estepa, 1998).

Entre otros errores puntuales, destacamos la falta de comprensión de la diferencia entre variable dependiente e independiente (Estepa, 1994), la confusión entre las dos rectas de regresión, la interpretación incorrecta de los coeficientes de regresión y de su relación con la pendiente de la recta y tipo de correlación (Sánchez, 1999). Otros estudiantes no diferencian los casos en que debe aplicarse o no una recta de regresión que cruce el origen de coordenadas (Eisenhaver, 2003). Agnelli, Konic, Peparelli y Flores (2009) indican que algunas de estas dificultades pueden estar ligadas al estudio previo de la función lineal, que se generaliza excesivamente.

\section{Investigaciones sobre libros de texto de matemáticas}

La investigación sobre libros de texto de matemáticas es amplia, siendo menor en estadística, donde encontramos ejemplos como los de Brewer (1985), Schacht (1990) o Cobo y Batanero (2004).

Respecto a la correlación y regresión destacamos dos investigaciones: en la primera de ellas, Sánchez Cobo (1999) estudia las definiciones relacionadas con la regresión en once libros de texto de bachillerato, publicados entre 1987 y 1990, clasificándolas según se presenten apoyadas en otros conceptos (de- 
finición estructural), se definan únicamente de forma procedimental, o bien la definición sea una mezcla de las anteriores. Por otro lado, Lavalle, Micheli y Rubio (2006) analizan la enseñanza de la correlación y regresión en siete textos, considerando los conceptos y procedimientos asociados, así como sus relaciones. Encuentran distintos niveles de profundidad en el tratamiento del tema. Solamente un texto define de modo estructural el concepto de recta de regresión y dos aluden a funciones de ajuste distintas a la lineal. En la mayoría se trata también la estimación de la variable dependiente.

Por su parte Gea, Batanero, Cañadas y Contreras (2013) identifican los problemas que permiten dotar de significado a la regresión en textos de bachillerato de Ciencias Sociales. Gea, Batanero, Fernandes y Gómez (2014) analizan, en estos mismos libros de texto, la presentación de la distribución bidimensional y sus representaciones gráfica y tabular. Gea, Batanero, Arteaga, Cañadas y Contreras (2014) estudian el lenguaje simbólico, expresiones algebraicas y verbales sobre la correlación y regresión. Queda pendiente el análisis del modo en que se utilizan los recursos tecnológicos en los textos mencionados, que es el objetivo del presente trabajo.

\section{Metodología}

Se analizaron 16 libros de texto, 8 en cada una de las modalidades de primer curso de bachillerato, publicados según la normativa vigente (MEC, 2007b). Los textos se eligieron por ser los más utilizados en la enseñanza pública en la Comunidad Autónoma de Andalucía y ser publicados por editoriales de gran tradición y prestigio. En el anexo 1 se presentan los libros analizados junto con un código utilizado a lo largo del trabajo; dicho código será $\mathrm{H} 1$, $\mathrm{H} 2$, etc., para los textos correspondiente a la modalidad en Humanidades y Ciencias So- ciales, y T1, T2, etc., para los textos dirigidos a la modalidad en Ciencia y Tecnología. Aquí se resalta el hecho de que todos han estado vigentes hasta la fecha. De cada editorial se tomaron los libros dirigidos a las dos modalidades de bachillerato citadas.

Los recursos tecnológicos se consideran en los textos de tres modos diferentes: 1) en el planteamiento o resolución de problemas, sobre todo en la descripción de procedimientos; 2) mediante referencias a recursos en internet; y 3) mediante un CD que se incluye en muchos de los textos con diverso contenido. Se realiza un análisis de estos usos del siguiente modo:

- En primer lugar se estudian los recursos tecnológicos que se incluyen en el desarrollo del tema, por ejemplo: direcciones de páginas web o applets, descripción de procedimientos para el uso de recursos tecnológicos como la calculadora, o propuesta de tareas en que se indique el uso de recursos tecnológicos para resolverlas o resueltas.

- Luego, se analiza el contenido del $C D$ que algunos textos incluyen como material complementario en la enseñanza y aprendizaje. Se observa el propósito de los archivos que se incluyen (descripción de recursos tecnológicos, propuesta de tareas, etc.) y los recursos tecnológicos incluidos en ellos (hojas de cálculo, calculadora, software, etc.).

El método usado es el análisis de contenido, que difiere de otras técnicas de estudio documental (por ejemplo, del método histórico), porque sustituye en lo posible las interpretaciones y subjetividad del estudio de documentos o de comunicaciones por procedimientos estandarizados, con el fin de convertir en datos los contenidos analizados (León y Montero, 2002). 


\section{Resultados y discusión}

A continuación se exponen los resultados del análisis respecto a los tres modos descritos de uso de recursos tecnológicos.

\section{Uso de recursos tecnológicos en la resolución de problemas y procedimientos}

En primer lugar se estudió el conjunto de problemas (y en general tareas) propuestos en el tema en cada uno de los textos, así como el conjunto de procedimientos descritos para resolverlos, entendiendo como tal técnicas de cálculo o estrategias introducidas para el análisis bidimensional. Es decir, las relacionadas con la representación gráfica o tabular de los datos bidimensionales; la estimación, cálculo o interpretación de la correlación y el cálculo y uso de la recta de regresión en la predicción.

La mayoría de los problemas (y los procedimientos asociados para resolverlos) se plantean sin mención explícita ni implícita a recursos tecnológicos (tabla 1). Ello puede deberse, en gran parte, a que los conjuntos de datos propuestos para realizar estas tareas son muy pequeños; de hecho, demasiado pequeños para obtener una estimación aceptable estadísticamente del coeficiente de correlación o la recta de regresión. No se sigue la recomendación de que los estudiantes trabajen la estadística con conjuntos de datos reales, según la normativa curricular (MEC, 2007b; MECD, 2015) y algunos investigadores como Hall (2011).

En el caso de las tareas o procedimientos donde se sugiere explícitamente al estudiante utilizar recursos tecnológicos, solo se encontró mención a la calculadora y la hoja de cálculo Excel, sin referencia a otro tipo de software o recurso. Ambos se mencionan de manera muy esporádica (tabla 1).

Tabla 1. Porcentaje de tareas según recurso tecnológico usado en los libros analizados

\begin{tabular}{lllllllll}
\hline & H1 & H2 & H3 & H4 & H5 & H6 & H7 & H8 \\
\hline Excel & & 21,7 & 0,8 & 4,4 & & & & 5,7 \\
\hline Calculadora & 4,9 & 2,3 & & & 4,7 & 2,3 & 4,2 & \\
\hline No usa & 95,1 & 76 & 99,2 & 95,6 & 95,3 & 97,7 & 95,8 & 94,3 \\
\hline Total tareas & 268 & 221 & 258 & 225 & 318 & 176 & 402 & 297 \\
\hline & $\mathrm{T} 1$ & $\mathrm{~T} 2$ & $\mathrm{~T} 3$ & $\mathrm{~T} 4$ & $\mathrm{~T} 5$ & $\mathrm{~T} 6$ & $\mathrm{T7}$ & $\mathrm{T}$ \\
\hline Excel & & 21,7 & 1,1 & 4,4 & & & & 6 \\
\hline Calculadora & 4,8 & 2,3 & & & 4,9 & 2,3 & 4,6 & \\
\hline No usa & 95,2 & 76 & 98,9 & 95,6 & 95,1 & 97,7 & 95,4 & 94 \\
\hline Total tareas & 269 & 221 & 186 & 225 & 307 & 176 & 371 & 284 \\
\hline
\end{tabular}

De esta investigación se sugiere aumentar las referencias al uso de Excel en los textos, puesto que su aprendizaje se menciona expresamente como objetivo en los documentos curriculares citados. Esta hoja de cálculo es de gran utilidad en el estudio de este tema, ya que dispone de herramientas muy completas y sencillas de usar para el análisis bidimensional, sobre todo para los contenidos matemáticos de correlación y regresión. Dichas herramientas son interactivas e 
incluyen, entre otras posibilidades: la representación gráfica del diagrama de dispersión, al que se puede cambiar la escala y añadir la línea de tendencia según diversos modelos (lineal, logarítmico, polinómico, exponencial); el cálculo de los parámetros de la función de ajuste, en cualquiera de los anteriores modelos; la obtención de valores de la variable dependiente, dado un valor de la independiente; la interpolación y extrapolación, y el cálculo del coeficiente de determinación para valorar la bondad del ajuste.

Hay diferencias notables en los libros, pues [H2] y [T2] apoyan el $24 \%$ de las situaciones que plantean en el uso de recursos tecnológicos, siendo los únicos textos que incluyen el uso tanto de la calculadora como de la hoja de cálculo Excel. El uso de estos recursos en el resto de textos está alrededor de un $5 \%$, salvo en [H3] y [T3] que solo llegan al $1 \%$ y en [H6] y [T6] con cerca de un $2 \%$. Por otro lado, [H3], [T3], [H4], [T4], [H8] y [T8] optan por el tratamiento de Excel dentro del tema, mientras que el resto de los textos (excepto los citados [H2] y [T2]) solo sugieren el uso de la calculadora.

El grado de detalle con que los textos describen los recursos tecnológicos al presentar los procedimientos en el desarrollo del tema es también variable. En los textos [H2], [T2], [H4], [T4], [H8] y [T8] se incluye una descripción detallada de los pasos necesarios para obtener el diagrama de dispersión, la covarianza o la recta de regresión con Excel (ver una parte de la descripción en la figura 1). Curiosamente, ninguno de ellos se refiere a la posibilidad de realizar otros gráficos bivariantes que puedan complementar la información del diagrama de dispersión mediante la hoja de cálculo; por ejemplo, un diagrama de barras tridimensional.

\section{3 | Cálculo de la recta de regresión}

Selecciona las celdas desde B3 a C8 y pulsa en 10 . Elige el tipo XY (Dispersión) y pulsa en Finalizar.

Selecciona los puntos de la gráfica obtenida pinchando con el ratón uno de ellos. A continuación pulsa en el botón derecho del ratón, y elige en la ventana emergente Agregar línea de tendencia.

Escoge Tipo linealy en la solapa de Opciones, marca presentar ecuación en el gráfico. Obtendrás lo siguiente:

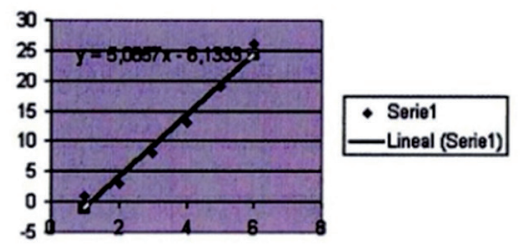

Figura 1. Descripción del análisis de datos bidimensional en Excel ([H4], p. 234)

Los textos [H2], [T2], [H3], [T3], [H8] y [T8] describen la forma de efectuar una predicción de la variable dependiente a partir de un valor de la independiente utilizando Excel (figura 2). Además, [H2] y [T2] son los únicos que incluyen también la descripción de estos procedimientos mediante Windows Calc (Linux).

\begin{tabular}{|c|c|c|c|c|c|c|c|c|c|}
\hline & A & B. & C & D & $E$ & $\mathrm{~F}$ & G & H & \\
\hline 25 & & & & PESO = & 0,4217 & TALLA + & & & \\
\hline 26 & & & & TAUA $=$ & 1,3701 & PESO + & & & \\
\hline 27 & Se es & sper: & & un niño que mide & 109.9 & $\mathrm{~cm}$ & pese & 20,13583 & $3] \mathrm{kg}$ \\
\hline 28 & Se es & spert & & e un nirio que pese & 20,14 & & mida & 109,906814 & $4 \mathrm{~cm}$ \\
\hline
\end{tabular}

Figura 2. Ejemplo de predicción a través de la hoja Excel ([T8], p. 407) 


\section{Referencias a recursos en Internet}

El uso de internet como recurso didáctico en probabilidad y estadística ha sido resaltado por muchos autores. Batanero (1998) citó, entre otros usos, el acceso a la información y a conjuntos de datos que se pueden observar en proyectos estadísticos, el trabajo a distancia de alumnos y profesores, el uso de programas de cálculo o simulación interactivos (applets), o la búsqueda de información complementaria sobre un tema.

Sada (2011) describe las características y potencialidades de los applets y sus posibilidades en el aula; sugiere que facilitan la comprensión, la representación e interpretación de gráficos estadísticos, la observación colectiva de resultados de un número elevado de simulaciones, a la vez que favorecen la dinámica del debate y el ritmo del aula. Arnaldos y Faura (2012) mencionan la importancia de las simulaciones interactivas incorporadas en la docencia, pues al poseer un formato más atractivo, permiten incidir de forma más directa en el proceso de aprendizaje del alumno. Contreras (2011) realiza un esbozo detallado de recursos tecnológicos para el caso de la probabilidad condicional y Reyes (2013) para el caso de la probabilidad en educación primaria; ambos estudios se fundamentaron en el mismo marco teórico que se utiliza en el presente trabajo.

Para el caso particular de la estadística bidimensional, hay muchos recursos en internet que pueden facilitar la comprensión del tema. Por ejemplo, se suele introducir a los estudiantes al criterio de mínimos cuadrados, es decir, elegir la recta de regresión de modo que haga mínima la suma de los cuadrados de las desviaciones de los puntos en el diagrama de dispersión a dicha recta. La deducción matemática formal de la ecuación de la recta de mínimos cuadrados es muy abstracta para los estudiantes. Sin embargo, es posible ofrecerles una justificación informal, del sentido del método de mínimos cuadrados con applets, por ejemplo, el disponible en el servidor de Educación de la Comunidad Autónoma de Navarra (//docentes.educacion.navarra.es/msadaall/geogebra/figuras/ e3regresion.htm). Como se observa en la figura 3, los estudiantes pueden usar los datos disponibles en este recurso o introducir sus propios datos, que quedan representados en un diagrama de dispersión, junto con el cuadrado de las desviaciones de cada punto a la recta de regresión. El programa permite buscar a ojo la recta de regresión y comparar con la recta de mínimos cuadrados. Es posible comprobar propiedades como que la recta de regresión pasa por el centro de gravedad (punto cuyas coordenadas son las medias muestrales de las dos variables). Por otro lado, como el recurso representa también el coeficiente de correlación lineal de Pearson, el estudiante puede experimentar cómo varía este coeficiente modificando la posición de los puntos en el diagrama y estudiar el efecto de la mayor o menor dispersión de la nube sobre este coeficiente. 


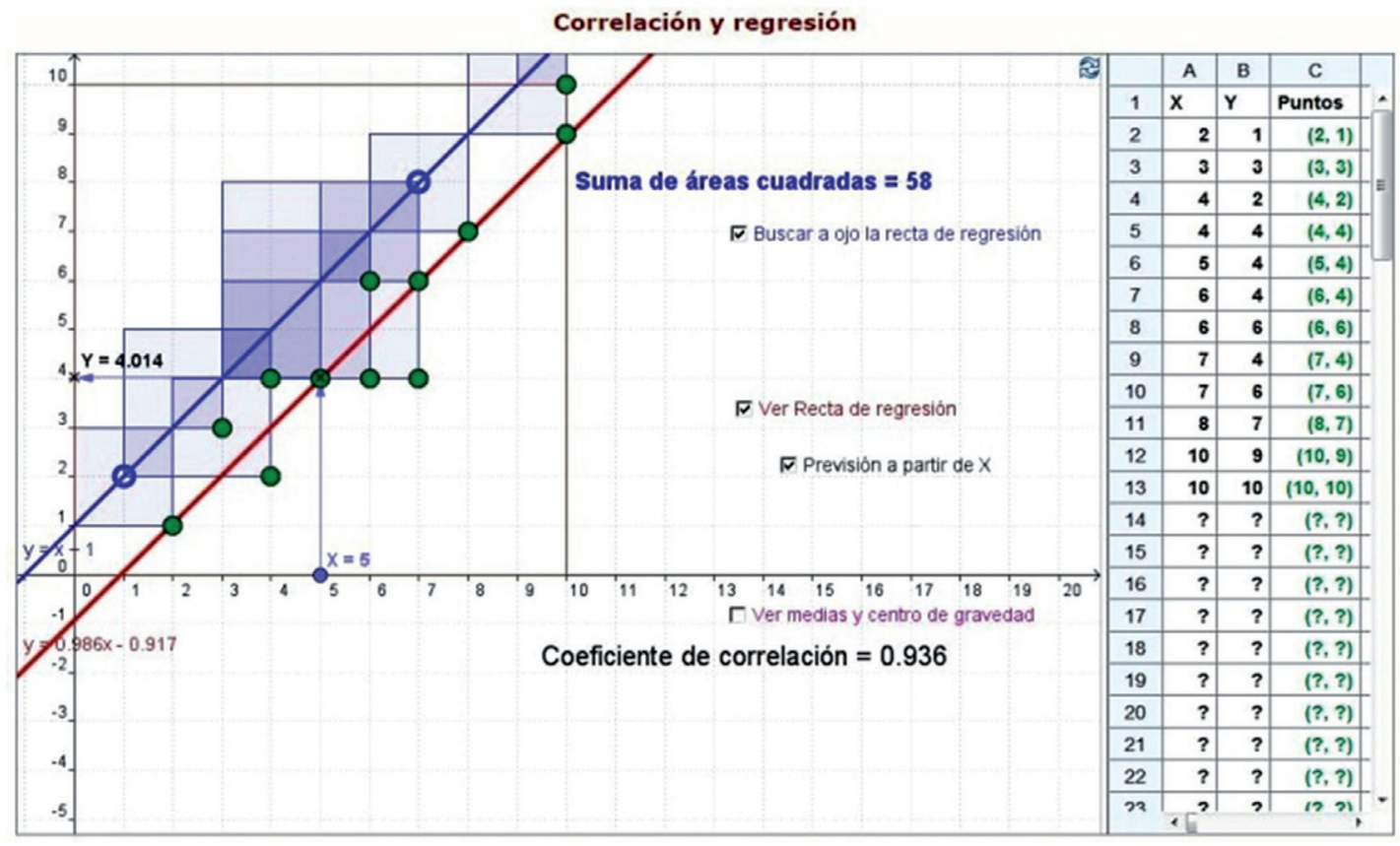

Figura 3. Applet sobre correlación y regresión en el servidor de Educación de Navarra

En la tabla 2 se presentan los resultados del análisis sobre referencias a recursos en internet en el tema de estadística bidimensional en los textos analizados. Allí se incluyen suge- rencias de uso de internet únicamente en la mitad de los textos estudiados ([H2], [T2], [H3], [T3], [H4], [T4], [H5] y [T5]), con poca variación en las dos modalidades de bachillerato.

Tabla 2. Recursos tecnológicos en los libros analizados

\begin{tabular}{lllllllll}
\hline & $\mathrm{H} 1$ & $\mathrm{H} 2$ & $\mathrm{H} 3$ & $\mathrm{H} 4$ & $\mathrm{H} 5$ & $\mathrm{H} 6$ & $\mathrm{H} 7$ & $\mathrm{H} 8$ \\
\hline Unidades didácticas y documentos teóricos & & $\mathrm{x}$ & $\mathrm{x}$ & $\mathrm{x}$ & $\mathrm{x}$ & & & \\
\hline Applets & & & $\mathrm{x}$ & $\mathrm{x}$ & & & \\
\hline & $\mathrm{T} 1$ & $\mathrm{~T} 2$ & $\mathrm{~T} 3$ & $\mathrm{~T} 4$ & $\mathrm{~T} 5$ & $\mathrm{~T} 6$ & $\mathrm{~T} 7$ & $\mathrm{~T} 8$ \\
\hline Unidades didácticas y documentos teóricos & & $\mathrm{x}$ & $\mathrm{x}$ & $\mathrm{x}$ & $\mathrm{x}$ & & & \\
\hline Applets & & & $\mathrm{x}$ & $\mathrm{x}$ & & & \\
\hline
\end{tabular}

Una observación más profunda reveló pocas indicaciones al uso de applets, y en general, de referencias a internet, a pesar de la importancia señalada por autores como Sada (2011). En [H3], [T3], [H5] y [T5] se encontraron más enlaces a internet que en $[\mathrm{H} 4]$ y $[\mathrm{T} 4]$, que contienen solo una referencia. En su mayoría, estos vínculos conducen a sitios web con unidades didácticas sobre la correlación y regresión, aunque en otros casos se describe también el uso de algún applet, alguna reflexión didáctica sobre una investigación basada en el análisis de la correlación, o una prueba de autoevaluación. En concreto, las referencias a internet en los textos son las siguientes:

- $\quad[H 3],[T 3],[H 4]$ y [T4] proporcionan un enlace a una unidad didáctica sobre correlación y regresión lineal denominada "Descartes 2D Estadística y probabilidad", que incluye 
actividades y aplicaciones interactivas (descartes.cnice.mec.es/materiales_didacticos/).

- [H3] y [T3] sugieren también el uso de una applet para analizar la correlación lineal, y la regresión lineal y cuadrática, que permite visualizar los datos en un diagrama de dispersión, pero no interactuar con ellos. También incluye una referencia a una aplicación con ejercicios de autoevaluación del aprendizaje (www.xtec.net/aulanet/ud/mates/ estadistica/tr3/index.htm).

- $\quad$ En [H5] y [T5] se encontraron los siguientes enlaces: a) un ejercicio que propone estudiar la relación entre la latitud y la temperatura media anval de un conjunto de ciudades, y permite al alumno completar estos datos con otros; b) otro ejercicio con datos reales sobre talla y el peso de 20 niños; c) una página web sobre cinemática, que incluye un applet interactivo (www.sc.ehu.es/sbweb/fisica/cinematica/regresion/regresion. $\mathrm{htm}$ ), el cual proporciona los valores de la pendiente y la ordenada en el origen de la recta de regresión por el método de mínimos cuadrados, el error de predicción, y el coeficiente de correlación lineal; d) un enlace a un texto sobre el origen de la correlación y regresión (www.iescarrus. com/edumat/taller/regresion/regresion_01.htm).

\section{CD con material tecnológico}

Para finalizar el estudio se analizó el contenido del CD que complementa a algunos libros de texto, en los cuales se encontraron los contenidos que se describen a continuación.

Descripción del uso de recursos tecnológicos. En estos CD se suelen incluir documentos que detallan la forma de llevar a cabo los procecimientos asociados a la correlación y regresión con ayuda de diferentes recursos tecnológicos como puede ser la calculadora gráfica $([\mathrm{Hl}]$ y $[\mathrm{T} 1])$, la hoja Excel $([\mathrm{H} 1],[\mathrm{Tl}]$, [H6] y [T6]) u otros programas. Es el caso de los textos [H1] y [T1] que describen el uso de Derive; o los textos [H2] y [T2] que incluyen el programa Wiris, con una rutina ya programada para tratar la estadística unidimensional y la regresión lineal. Además, $[\mathrm{H} 1]$ y $[\mathrm{T} 1]$ son los únicos textos que ponen a disposición del estudiante una hoja de cálculo (figura 4), en la que, a partir de datos en un listado, se puede obtener las tablas de doble entrada, el diagrama de dispersión, la covarianza, la correlación, y la recta de regresión.

Complementos teóricos. Algunos CD incluyen enlaces a unidades didácticas de apoyo ([H5], [T5], [H8] y [T8]) o documentos biográficos sobre los autores que crearon la regresión y correlación. En [H6] y [T6] también se incluyen algunas tareas de correlación y regresión propuestas en la prueba de acceso a la universidad (selectividad), con documentos de lectura asociados. Además [H6] y [T6] repiten el contenido del tema del libro, aunque utilizando animación e hipertexto. 


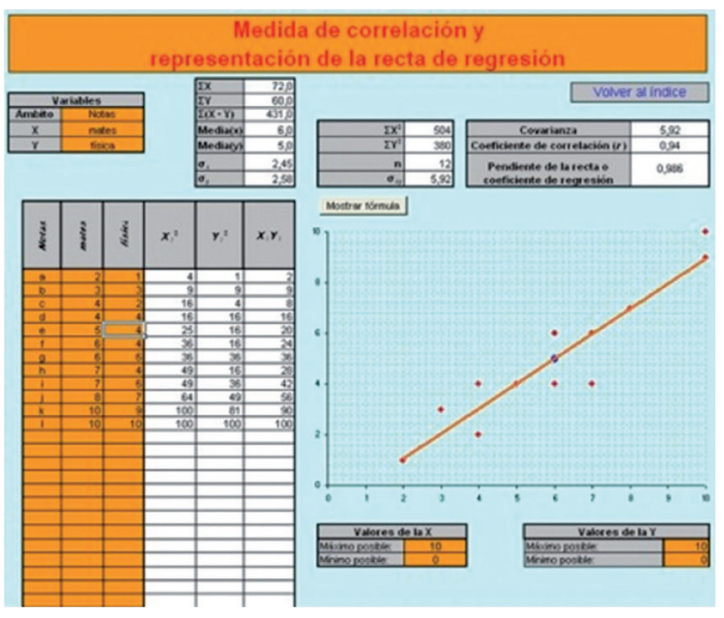

Figura 4. Hoja de cálculo incluida en el CD en $[\mathrm{Hl}]$

Actividades. Algunos CD que acompañan a los textos incluyen actividades con diversa finalidad. Así, en [H1] y [T1] se incluye la solución de la prueba de autoevaluación del tema. [H6] y [T6] proponen dos tipos de tareas: unas incluidas en el documento que describe el uso de la hoja Excel, y otras actividades de evaluación interactivas, en formato de opción múltiple, en las que, una vez que el estudiante elige su solución, el programa proporciona la correcta. Las tareas interactivas incluidas se clasifican en tres tipos:

a. Asociar un diagrama de dispersión a valores del coeficiente de correlación, y análisis de las distribuciones marginales.

b. Covarianza, correlación y estimación de sus valores.

C. Regresión y predicción.

[H5] y [T5] proponen tareas de matemáticas recreativas, como crucigramas o juegos de memoria. [H7] y [T7] incluyen tareas resueltas sobre construcción de una tabla de doble entrada, cálculo del coeficiente de correlación a partir de la tabla, determinación de la media, signo del coeficiente de correlación, y esti- maciones a partir de las rectas de regresión. También incluye un video donde un profesor explica la resolución, en una pizarra, de las tareas. [H8] y [T8] incluyen tres tareas interactivas: en la primera se debe elegir una recta de regresión bajo indicaciones dadas; en la segunda se deben organizar unos datos, y en la tercera se debe asignar un valor del coeficiente de correlación a diferentes diagramas de dispersión (figura 5).

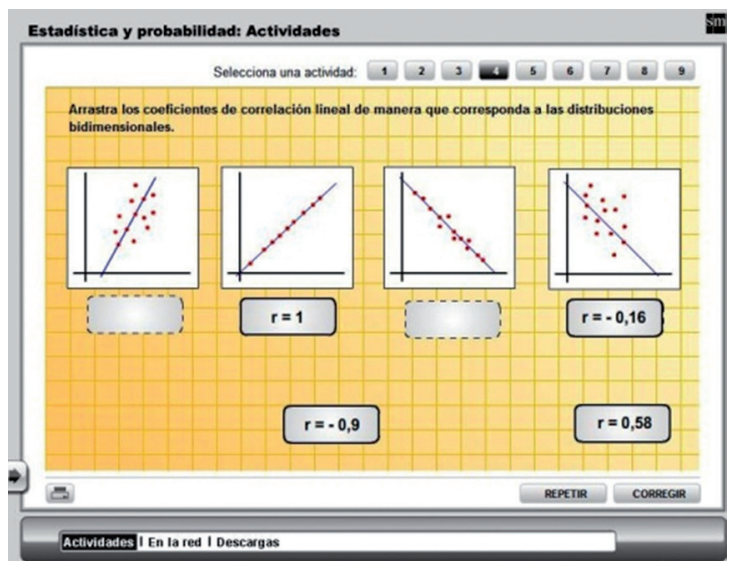

Figura 5. Tareas propuestas en el CD de [H8]

Enlaces a programas y descarga de los mismos. Algunos CD muestran enlaces para la descarga de determinados programas como Derive, Ecuv 2.3.33 o Graph.

Otros enlaces. [H8] y [T8] contienen una sección denominada "En la red", desde la que se accede a diferentes páginas de internet sobre el origen de la estadística, talleres de estadística, tipos de encuestas y enlaces de descarga de datos; por ejemplo, del Ministerio de Empleo y Seguridad Social en España (http://www.sepe.es/contenidos/intermedia. html).

En la tabla 3 se resume el análisis realizado sobre el contenido del CD, excepto la parte de applets que se comentó y describió en la tabla 2. Hay bastante variedad en los 
textos, como se observa en el caso de [H1] y [ $\mathrm{T} 1]$ que son los más completos en cuanto a descripción de uso de diferentes recursos tecnológicos en el tema.

Además, la mitad de los textos incluyen actividades interactivas de evaluación, pero el resto de categorías aparecen con baja frecuencia. Si se añaden los textos [H3], [T3], [H4] y [T4], que incluían enlaces a applets (tabla 2), aparentemente la mayoría de los textos incluyen actividades interactivas, pero de hecho, los marcados en la tabla 3 son simplemente actividades de autoevaluación y no de exploración.

Tabla 3. Recursos tecnológicos en los CD que acompañan a los textos

\begin{tabular}{|c|c|c|c|c|c|c|c|c|}
\hline & $\mathrm{Hl}$ & $\mathrm{H} 2$ & $\mathrm{H} 3$ & $\mathrm{H} 4$ & $\mathrm{H} 5$ & H6 & $\mathrm{H7}$ & $\mathrm{H} 8$ \\
\hline Descripción análisis bidimensional con Excel & $\mathrm{x}$ & & & & & $\mathrm{x}$ & & \\
\hline Descripción análisis bidimensional con Derive & $x$ & & & & & & & \\
\hline Análisis bidimensional con calculadora gráfica & $x$ & & & & & & & \\
\hline Actividades interactivas de evaluación & & & & & $x$ & $x$ & $x$ & $\mathrm{x}$ \\
\hline Hoja de cálculo: Tabla de doble entrada; correlación y regresión & $x$ & & & & & & & \\
\hline Enlaces a programas de cálculo & & $x$ & & & $x$ & & & \\
\hline \multirow[t]{2}{*}{ Enlaces a otras páginas web } & & & & & $\mathrm{x}$ & & & $\mathrm{x}$ \\
\hline & $\mathrm{T1}$ & T2 & T3 & T4 & T5 & T6 & $\mathrm{T7}$ & T8 \\
\hline Descripción análisis bidimensional con Excel & $\mathrm{x}$ & & & & & $\mathrm{x}$ & & \\
\hline \multicolumn{9}{|l|}{ Descripción análisis bidimensional con Derive } \\
\hline Análisis bidimensional con calculadora gráfica & $\mathrm{x}$ & & & & & & & \\
\hline Actividades interactivas de evaluación & & & & & $x$ & $x$ & $x$ & $x$ \\
\hline Hoja de cálculo: tabla de doble entrada; correlación y regresión & $\mathrm{x}$ & & & & & & & \\
\hline Enlaces a programas de cálculo & & $x$ & & & $x$ & & & \\
\hline Enlaces a otras páginas web & & & & & $\mathrm{x}$ & & & $\mathrm{x}$ \\
\hline
\end{tabular}

Al examinar el tipo de correlación presentado en estas actividades interactivas incluidas en los CD, también se encontró variedad: las propuestas en [H6] y [T6] presentan, en su mayoría, una correlación alta entre las variables, aunque en las aplicaciones de la correlación es frecuente observar correlaciones moderadas. En [H8] y [T8] hay más variedad de intensidad de la correlación, pues de hecho se incluyen actividades con dependencia funcional entre las variables. Por otra parte, en [H6] y [T6] se proponen actividades donde predomina la dependencia directa, mientras que en [H8] y [T8] hay tantas tareas con dependencia directa como inversa. Mayoritariamente se trata el ajuste lineal entre las variables, aunque en [H6] y [T6] se propone una tarea bajo un ajuste polinómico de grado dos.

El resto del contenido de los CD se limita a reproducir el texto o incluir nuevos ejercicios de tipo tradicional, con pocos datos, los cuales no invitan a que el estudiante complete el ciclo de investigación descrito por Wild y Pfannkuch (1999).

\section{Conclusiones}

Este análisis sobre el uso o la referencia a recursos tecnológicos en los libros de texto para el tema de estadística bidimensional indica, en primer lugar, que su presencia dentro del mismo texto es muy escasa, ya que pocas veces las tareas o procedimientos se presentan teniendo en cuenta los recursos tecnológicos. 
Ello implica, de manera indirecta, que los conjuntos de datos utilizados en los ejercicios y tareas son pequeños, lo que tiene una doble consecuencia: a) las estimaciones de la correlación y regresión serán poco fiables, y b) un empobrecimiento de las aplicaciones de la correlación y regresión mostrada en los textos, al no poder trabajar con datos reales, como recomienda Hall (2011).

La situación mejora un tanto cuando se incluye el análisis del CD que acompaña al texto, pero no todo lo deseable. Por un lado, se asume que el uso del CD no será tan amplio como el del libro, pues requiere que el estudiante tenga el dispositivo de lectura, situación no muy común en las aulas. Por otro lado, hemos visto que el contenido del $C D$, en muchos casos, reproduce el del texto, salvo animaciones, o solo incluye referencias a lecturas históricas o actividades teóricas de autoevaluación del aprendizaje.

A pesar de la gran variabilidad en las editoriales en cuanto al uso de recursos tecnológicos en el tema de estadística bidimensional, en general, se prima más el refuerzo teórico que las sugerencias para que el estudiante trabaje el tratamiento de datos mediante la manipulación interactiva con la calculadora, hoja de cálculo, applets u otro software. En ese aspecto, destacamos los textos [H1] y [ $\mathrm{T} 1]$ pues incluyen hojas de cálculo programadas en Excel para el tratamiento de datos bidimensionales y análisis de la correlación y regresión.

Estos resultados son preocupantes ya que los estudiantes de primer curso de bachillerato provienen, en su mayoría, de la enseñanza secundaria, donde se promueve el desarrollo de competencias básicas como el "Tratamiento de la información y competencia digital" y la "Competencia matemática". En este sentido, existe una desconexión en los propósitos de desarrollo de estas competencias en los estudiantes entre estas etapas educativas.

En la etapa educativa de secundaria obligatoria, las directrices curriculares indican, para el desarrollo de la competencia matemática, la importancia de interpretar y expresar con claridad información mediante herramientas de apoyo adecuadas y a través del conocimiento matemático a situaciones de la vida (MEC, 2007a); y cómo disponer de información en bruto no produce conocimiento, sino que se requieren destrezas de razonamiento para organizarla, relacionarla, analizarla, sintetizarla y hacer inferencias y deducciones: "Ser competente en la utilización de las tecnologías de la información y la comunicación como instrumento de trabajo intelectual incluye utilizarlas en su doble función de transmisoras y generadoras de información y conocimiento" (p. 688).

Toda esta reflexión lleva a sugerir la necesidad de mejorar la presentación de los recursos tecnológicos en este tema en los libros de texto, pues la nueva normativa curricular (MECD, 2015) establece, para el último curso de la etapa de secundaria obligatoria (estudiantes de entre 14 y 15 años), en las dos especialidades de matemáticas dirigidas a las enseñanzas aplicadas o enseñanzas académicas, la introducción a la correlación a través del análisis de diagramas de dispersión o nubes de puntos.

En consecuencia, se recomienda al profesor reforzar los textos, respecto al uso de recursos tecnológicos, teniendo en cuenta su alta idoneidad didáctica en la enseñanza, no solo desde el componente afectivo, pues estos recursos son muy motivadores para el estudiante. Además, al facilitar el aprendizaje y la creatividad se refuerza la componente 
cognitiva; el poder ampliar las aplicaciones del tema lleva a conectar las matemáticas con otras materias (Contreras, 2011).

Agradecimientos

Proyecto EDU2013-41141-P (MEC) y grupo FQM126 (Junta de Andalucía).

\section{Referencias bibliográficas}

Agnelli, H.; Konic, P.; Peparelli, N.Z. y Flores, P. (2009). La función lineal obstáculo didáctico para la enseñanza de la regresión lineal. Revista Iberoamericana de Educación Matemática, 17, 52-61.

Arnaldos, F. y Faura, U. (2012). Aprendizaje de los fundamentos de la probabilidad apoyado en las TIC. @tic. revista d'innovació educativa, 9, 131-139

Batanero, C. (1998). Recursos para la educación estadística en Internet. Uno, 15, $13-26$.

Batanero, C. y Díaz, C. (2011). Estadística con proyectos. Granada: Universidad de Granada.

Batanero, C.; Godino, J.D. y Estepa, A. (1998). Building the meaning of statistical association through data analysis activities (Research Forum). En: A. Olivier y K. Newstead (eds.). Proceedings of the $22^{\text {nd }}$ Conference of the Internacional Group for the Psychology of Mathematics Education (pp. 221-236). Stellembosch, Sudáfrica: Universidad de Stellenbosh.

Biehler, R. (1997). Software for learning and for doing statistics. International Statistical Review 65(2), 167-190.

Brewer, J.K. (1985). Behavioral statistics textbooks: Source of myths and misconceptions? Journal of Educational and Behavioral Statistics 10(3), 252-268.

Chapman, L.J. y Chapman, J.P. (1967). Genesis of popular but erroneous psychodiagnostic observations. Journal of Abnormal Psychology 72(3), 193-204.

Chevallard, Y. (1991). La transposición didáctica. Del saber sabio al saber enseñado. Buenos Aires: Aique.

Cobo, B. y Batanero, C. (2004). Significados de la media en los libros de texto de secundaria. Enseñanza de las Ciencias 22(1), 5-18.

Consejería de Educación. (2008). Orden de 5 de agosto de 2008, por la que se desarrolla el currículo correspondiente al Bachillerato en Andalucía. Sevilla.

Contreras, J.M. (201 1). Evaluación de conocimientos y recursos didácticos en la formación de profesores sobre probabilidad condicional. Tesis Doctoral. Granada: Universidad de Granada. 
Cordero, F. y Flores, R. (2007). El uso de las gráficas en el discurso matemático escolar. Un estudio socioepistemológico en el nivel básico a través de los libros de texto. Revista Latinoamericana de Matemática Educativa 10(1), 7-38.

Eisenhaver, J.G. (2003). Regression through the origin. Teaching Statistics 25(3), 76-80.

Engel, J. y Sedlmeier, P. (201 1). Correlation and regression in the training of teachers. En: C. Batanero, G. Burrill y C. Reading (eds.). Teaching statistics in school mathematics-challenges for teaching and teacher education: A Joint ICMI/IASE study (pp, 247-258). Nueva York: Springer.

Estepa, A. (1994). Concepciones iniciales sobre la asociación estadística y su evolución como consecuencia de una enseñanza basada en el uso de ordenadores. Tesis doctoral no publicada. Granada: Universidad de Granada.

Estepa, A. (2008). Interpretación de los diagramas de dispersión por estudiantes de Bachillerato. Enseñanza de las Ciencias 26(2), 257-270.

Estepa, A. y Batanero, C. (1995). Concepciones iniciales sobre la asociación estadística. Enseñanza de las Ciencias 13(2), 155-170.

Fernandes, J.A.; Batanero, C.; Contreras, J.M. y Díaz, C. (2009). A simulação em Probabilidades e Estatística: potencialidades e limitações. Quadrante 18(1), 161-183.

Gea, M.M. (2014). Evaluación y desarrollo del conocimiento matemático para la enseñanza de la probabilidad en futuros profesores de educación primaria. Tesis doctoral no publicada. Universidad de Granada.

Gea, M.M.; Batanero, C.; Cañadas, G.R. y Contreras, J.M. (2013). Un estudio empírico de las situaciones-problema de correlación y regresión en libros de texto de bachillerato. En: A. Berciano, G. Gutiérrez, A. Estepa y N. Climent (eds.). Investigación en Educación Matemática XVII (pp. 293-300). Bilbao: Sociedad Española de Investigación en Educación Matemática (SEIEM).

Gea, M.M.; Batanero, C.; Arteaga, P.; Cañadas, G.R., Contreras, J.M. (2014). Análisis del lenguaje sobre la correlación y regresión en libros de texto de bachillerato. Suma, 76, 47-45.

Gea, M.M.; Batanero, C.; Fernandes, J.A. y Gómez, E. (2014). La distribución de datos bidimensionales en los libros de texto de matemáticas de bachillerato. Quadrante 23(2), 147-172.

Godino, J.D. (2002). Un enfoque ontológico y semiótico de la cognición matemática. Recherches en Didactique des Mathématiques 22(2-3), 237-284.

Godino, J.D. y Batanero, C. (1998). Funciones semióticas en la enseñanza y aprendizaje de las matemáticas. En: I. Vale y J. Portela (eds.). IX Reunión de la Sociedad Portuguesa de Investigación en Educación Matemática (SIEM) (pp. 47-62). Sociedad Portuguesa de Investigación en Educación Matemática: Guimaraes (Portugal).

Godino, J.D.; Batanero, C. y Font, V. (2007). The onto-semiotic approach to research in Mathematics education. ZDM. The International Journal on Mathematics Education 39(1-2), 127-135.

Hall, J. (201 1). Engaging teachers and students with real data: benefits and challenges. En: C. Batanero, G. Burrill, y C. Reading (eds.). Teaching statistics in school mathematics. Challenges for teaching and teacher education. A joint ICMI and IASE study (pp. 335-346). Nueva York: Springer. 
Herbel, B.A. (2007). From intended curriculum to written curriculum: Examining the "voice" of a mathematics textbook. Journal for Research in Mathematics Education 38(4), 344-369.

Lavalle, A.L.; Micheli, E.B. y Rubio, N. (2006). Análisis didáctico de regresión y correlación para la enseñanza media. Revista Latinoamericana de Investigación en Matemática Educativa 9(3), 383-406.

León, O.G. y Montero, I. (2002). Métodos de Investigación. Madrid: Mc Graw-Hill.

McKenzie, C.R.M. y Mikkelsen, L.A. (2007). A Bayesian view of covariation assessment. Cognitive Psychology 54(1), 33-61.

Ministerio de Educación y Ciencia (MEC) (2007a). Real Decreto 1631/2006, de 29 de diciembre, por el que se establecen las enseñanzas mínimas correspondientes a la Educación Secundaria Obligatoria. Madrid.

Ministerio de Educación y Ciencia (MEC) (2007b). Real Decreto 1467/2007, de 2 de noviembre, por el que se establece la estructura de Bachillerato y se fijan sus enseñanzas mínimas. Madrid: Autor.

Ministerio de Educación, Cultura y Deporte (MECD) (2015). Real Decreto 1 105/2014, de 26 de diciembre. Por el que se establece el currículo básico de la Educación Secundaria Obligatoria y del Bachillerato. Madrid.

Moritz, J. (2004). Reasoning about covariation. En: D. Ben-Zvi y J. Garfield (eds.). The challenge of developing statistical literacy, reasoning and thinking (pp. 221 255). Dordrecht: Kluwer.

Pratt, D.; Davies, N. y Connor, D. (2011). The role of technology in teaching and learning statistics, En: C. Batanero, G. Burrill y C. Reading (eds.). Teaching statistics in school mathematics. Challenges for teaching and teacher education. A joint ICMI and IASE study (pp. 97-107). Nueva York: Springer.

Reyes, K. (2013). Análisis de recursos en internet para la enseñanza de la probabilidad en la educación primaria. Tesis de máster no publicada. Granada: Universidad de Granada.

Rocha, P. y Gallego, A.P. (2009). Las prácticas docentes de los profesores de probabilidad y estadística en las facultades de ingeniería. Tecné, Episteme y Didaxis, (Extraordinario), 197-202.

Sada, M. (201 1). Los applets para la enseñanza de la estadística y probabilidad. Uno, 58, 38-48.

Sánchez C., F.T. (1999). Significado de la correlación y regresión para los estudiantes universitarios. Tesis doctoral no publicada. Granada: Universidad de Granada.

Schacht, S.P. (1990). Statistics textbooks: Pedagogical tools or impediments to learning? Teaching Sociology, 3, 390-396. 
Wild, C.J. y Pfannkuch, M. (1999). Statistical thinking in empirical enquiry. International Statistical Review 67(3), 223-265.

Zieffler, A.S. (2006). A longitudinal investigation of the development of college students' reasoning about bivariate data during an introductory statistics course. Tesis doctoral. Minnesota: Universidad de Minnesota.

Zieffler, A. y Garfield, J. (2009). Modeling the growth of students' covariational reasoning during an introductory statistics course. Statistics Education Research Journal 8(1), 7-31.

\section{Para citar este artículo:}

Gea, M. M., Batanero, C., López-Martins, M. M., Contreras, J. M. (2015) Los recursos tecnológicos en la estadística bidimensional en los textos españoles de bachillerato. Revista de la Facultad de Ciencia y Tecnología - Tecné, Episteme y Didaxis, (38), 113-132.

\section{Anexo 1. Textos utilizados en el análisis}

[H1]. Colera, J.; Oliveira, M.J.; García, R. y Santaella, E. (2008). Matemáticas aplicadas a las Ciencias Sociales I. Madrid: Grupo Anaya.

[H2]. Arias, J.M. y Maza, I. (201 1). Matemáticas aplicadas a las Ciencias Sociales 1. Madrid: Grupo Editorial Bruño.

[H3]. Anguera, J.; Biosca, A.; Espinet, M.J.; Fandos, M.J.; Gimeno, M. y Rey, J. (2008). Matemáticas I aplicadas a las Ciencias Sociales. Barcelona: Guadiel - Grupo Edebé.

[H4]. Monteagudo, M.F. y Paz, J. (2008). 1. Bachillerato. Matemáticas aplicadas a las Ciencias Sociales. Zaragoza: Edelvives (Editorial Luis Vives).

[H5]. Martínez, J.M.; Cuadra, R. y Heras, A. (2008). Matemáticas aplicadas a las Ciencias Sociales. 1. ${ }^{\circ}$ Bachillerato. Madrid: McGraw-Hill.

[H6]. Bescós, E. y Pena, Z. (2008). Matemáticas aplicadas a las Ciencias Sociales. Vizcaya: Oxford University Press España.

[H7]. Antonio, M.; González, L.; Lorenzo, J.; Molano, A.; Del Río, J.; Santos, D. y de Vicente, M. (2009). Matemáticas aplicadas a las Ciencias Sociales I. Madrid: Santillana Educación.

[H8]. Vizmanos, J.R.; Hernández, J.; Alcaide, F.; Moreno, M. y Serrano, E. (2008). Matemáticas aplicadas a las Ciencias Sociales 1. Madrid: Ediciones SM.

[T1]. Colera, J.; Oliveira, M.J.; García, R. y Santaella, E. (2008). Matemáticas I. Madrid: Grupo Anaya.

[T2]. Arias, J.M. y Maza, I. (201 1). Matemáticas 1. Madrid: Grupo Editorial Bruño.

[T3]. Biosca, A.; Doménech, M.; Espinet, M.J.; Fandos, M.J. y Jimeno, M. (2008). Matemáticas I. Barcelona: Guadiel - Grupo Edebé.

[T4]. Monteagudo, M.F. y Paz, J. (2008). 1. B Bachillerato. Matemáticas. Ciencias y Tecnología. Zaragoza: Edelvives (Editorial Luis Vives). 
[T5]. Martínez, J.M.; Cuadra, R. y Barrado, F.J. (2007). Matemáticas 1. Bachillerato. Madrid: McGraw-Hill.

[T6]. Bescós, E. y Pena, Z. (2009). Matemáticas. 1. Bachillerato. Navarra: Oxford University Press España.

[T7]. Antonio, M.; González, L.; Lorenzo, J.; Molano, A.; Del Río, J.; Santos, D. y de Vicente, M. (2008). Matemáticas I. 1. Bachillerato. Madrid: Santillana Educación.

[T8]. Vizmanos, J.R.; Hernández, J.; Alcaide, F.; Moreno, M. y Serrano, E. (2008). Matemáticas 1. Madrid: Ediciones SM. 